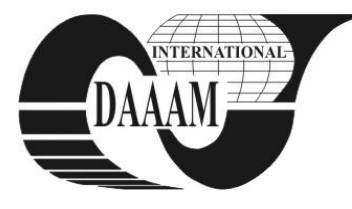

Annals of DAAAM for 2011 \& Proceedings of the 22nd International DAAAM Symposium, Volume 22, No. 1, ISSN 1726-9679 ISBN 978-3-901509-83-4, Editor B. Katalinic, Published by DAAAM International, Vienna, Austria, EU, 2011 Make Harmony between Technology and Nature, and Your Mind will Fly Free as a Bird Annals \& Proceedings of DAAAM International 2011

\title{
CONTROL UNIT FOR HUMANOID ROBOT
}

\author{
FLORIAN, T[omas]; BURIAN, F[rantisek] \& ZALUD, L[udek]
}

\begin{abstract}
The aim of this paper was to get acquainted with a commercional kit of a humanoid robot Robonova-I, study a manufacturer-programmed control and robot's potency, find out a gear mechanism (robotic servos), design an enhanced model of the control unit, design a printed circuit board (PCB) and occupy and energize it and pre-set a software for the control by means of PC and a serial line.

Key words: microcontroller, Robonova-I, servo, humanoid robot, control unit
\end{abstract}

\section{ROBONOVA-I}

Robonova-I is a model of a humanoid robot that is $31.8 \mathrm{~cm}$ high and weighs $1.33 \mathrm{~kg}$. It is powered by 16 servomotors and its manufacturer is Korean company Hitec Robotics. There are five servomotors for each leg and three for each arm. For comfortable controlling the robot is operated by an enclosed IR controller. By pressing the button you initiate the subprogram that was allocated to this number in the main program. There is a variety of robot's modification (plenty of nonofficial upgrades are situated on the internet) and you can also add further components (gyroscope, accelerometer, ultrasonic range finder, camera, faster servomotor, LCD display, voice module, bluetooth, radio module for controlling, etc.)

\section{THE CONTROL UNIT}

The control unit cares for the robot control and communication with all the devices connected and it is occupied by ATmega128 microcontroller by ATMEL company. The motherboard contains 24 signal pins for connecting of servomotors, 8 channels of A/D converter, 3 PWM outputs (pulse width modulation) and I2C connector for additional devices. Further the following devices are connected: a piezo converter for sound effects, a connector for LED diode (situated in the head of the robot), a power, an on/off switch, a connector for connection to PC via a serial cable (RS-232).

\section{DIGITAL ROBOTIC SERVO}

The robot is controlled by means of robotic servomotors labelled HSR-8498HB manufactured by Hitec company. Each servo is occupied by ATmega8 microcontroller by ATMEL company.

\subsection{Servomotor}

Servomotor (servo) is a motor with an integrated gear unit, a location sensor (often a potentiometer) and a control electronics that has three outlets - a power, a ground and a control signal. Servos usually have a rotation range (typically $180^{\circ}$ or $120^{\circ}$ ). The rotation is controlled by means of the pulse width that is received via a control line wire. Servos are most widely used in modelling industry.

\subsection{HMI protocol}

HMI protocol includes three modes (Ibbotson, 2007):

1. Standard mode $(550-2450 \mu \mathrm{s})$

2. Enhanced mode $(50 \mu \mathrm{s})$

3. Serial mode $(416 \mu \mathrm{s})$

Each signal works in a different segment of time therefore no initialisation or its identification are needed.

\subsubsection{Standard mode}

Servo works with signals of 550-2450 $\mu$ s length range through which we set its position. If it receives a signal outside this range, it will not react by the change of its position. The centre of servo is $1500 \mu \mathrm{s}$. In this mode the servo continually needs to be sent a position that it has to set, otherwise the loss of turning moment happens.

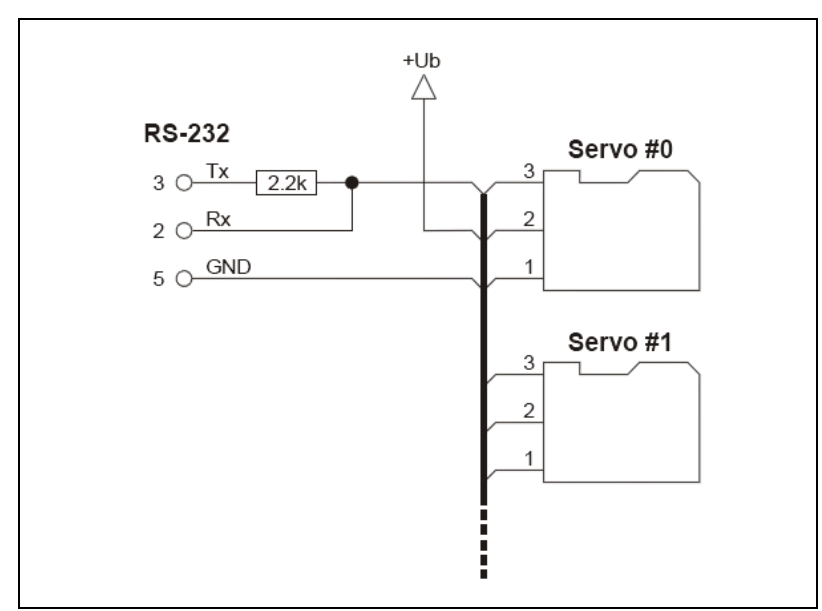

Fig. 1. Connection for serial communication (HSR Serial Communication, 2007)

\subsubsection{Enhanced mode}

The enhanced mode is specially created for needs of Robonova. In this mode the position is read from the servo.

\subsubsection{Serial mode}

In this mode we can connect and control up to 128 servos to the controller via one signal line wire. The serial line is used as an interface here. The only thing that has to be done before the connection of more servos is their initialisation. The most important is to set each servo a unique ID numer (from 0 to 127). This conception has finally been chosen for the design of the control unit.

\section{THE DESIGN OF A NEW CONTROL UNIT}

\subsection{Microcontroller}

The control unit was occupied by ColdFire microcontroller, version 2 core, by Freescale company. It concerns 32bit microcontroller of RISC type with a clock frequency of 60 
$\mathrm{MHz}$ and a large variety of peripheries (Freescale Semiconductor).

\subsection{Communication with the environment and devices connected}

To enable the unit to communicate with the environment and get information from it, the circuit board contains following components: I2C bus, 3xUART (one for servos, one for the control and one that is free), ethernet and an xBee module for wireless communication. Further devices connected are an accelerometer, a ultrasonic range finder, LED diodes for area illumination, a wireless camera for image and sound transmission to the operator.

\subsection{Control}

For wireless control we use the xBee module that works in unlicenced band of $2.4 \mathrm{GHz}$ and it is used for industrial applications. Its advantages are size, price and support from the manufacturer. For reasons given it is possible to use it without large checks on sample programs.

\subsection{Camera}

To allow the operator to monitor the environment in which the robot moves and to navigate it properly, it was necessary to use the wireless camera. We accentuate a low weight and size.

\subsection{Distance measurement}

We implemented a ultrasonic range finder SRF10 on the head of the robot to measure the distance. It is connected to the microcontroller via I2C bus (Everett, 1995). The measuring range varies from $3 \mathrm{~cm}$ to $6 \mathrm{~m}$.

\subsection{Acceleration sensor}

We implemented a three-axis accelerometer (by Freescale company labelled MMA7260QT) on PCB to protect and monitor robot's movement. It is used to protect sensitive parts of the robots at crash - a head tilting (to avoid damage of a camera, LED diodes or a distance sensor), arms setting or for continuity of movement.

\subsection{Comparison of original and newly designed conception}

\begin{tabular}{|l|l|l|}
\hline \multicolumn{1}{|c|}{ Equipment of units } & \multicolumn{1}{|c|}{ Original } & \multicolumn{1}{c|}{ Newly designed } \\
\hline Microcontroller & ATmega128 & ColdFire V2 \\
\hline Frequency & $16 \mathrm{MHz}$ & $60 \mathrm{MHz}$ \\
\hline Architecture & $8 \mathrm{bit}, \mathrm{RISC}$ & $32 \mathrm{bit}, \mathrm{RISC}$ \\
\hline Number of ADC & 8 & 8 \\
\hline Control & IR, controller & xBee, 2.4 GHz \\
\hline Max. number of servos & 24 & 128 \\
\hline Further buses & I2C & I2C, UART, ethernet \\
\hline Connected devices & $\begin{array}{l}\text { piezo } \\
\text { converter }\end{array}$ & $\begin{array}{l}\text { 2x LED, } \\
\text { accelerometer, } \\
\text { ultrasonic range } \\
\text { finder, wireless } \\
\text { camera }\end{array}$ \\
\hline
\end{tabular}

Tab. 1. Comparison of original and newly designed conception

\section{PROGRAM FOR PC CONTROL}

Communication through a serial line is not user friendly enough and the operator could be lost in a large amount of information about positions of each servo. Therefore we created a program (Liberty \& MacDonald, 2008) whose aim was to visualize data from the robot and allow its control.

The program was designed to allow clear and comfortable control of the whole robot and devices connected. It displays a help text that activates by rolling your mouse cursor for a number of elements. In menu there is an option to choose a connection to any COM port to which a wireless module is connected and start searching of the robot. The image from the camera opens as a separate window after pressing the button.

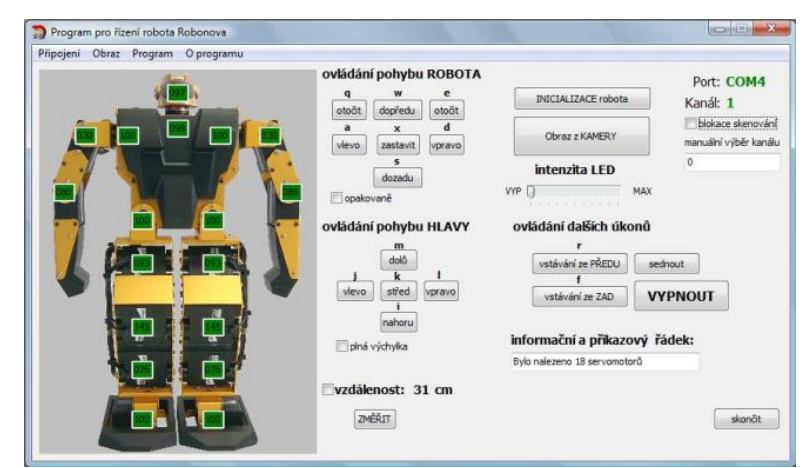

Fig. 2. The main program menu for the control of the robot

Pre-defined commands can be sent to the robot through key buttons. They concern movements forward, backward, left, right, turning left and turning right. All these commands have also their cyclic variations that repeat until the end command comes. All key buttons have their hot keys that are above and the command is also done by pressing them on the keyboard. Cyclic variations differ from the standard commands by the capital letter.

\section{CONCLUSION}

The main reason of our own conception of electronics is greater support of connectable devices, faster processor and more effective program for the control of the robot. The designed processor has much higher power, several times larger RAM and EEPROM (Flash) memory and greater amount of integrated circuits (UART, ethernet, timer, AD converter).

The project has been finished and it is fully functional. Further work on the robot has been assumed at Faculty of Electrical Engineering and Communication Brno University of Technology.

\section{ACKNOWLEDGEMENTS}

This project was supported by the Ministry of Education of the Czech Republic under Project 1M0567.

This work was supported by grant „Research of Modern Methods and Approaches in Automation" from the Internal Grant Agency of Brno University of Technology (grant No. FEKT-S-11-6)

\section{REFERENCES}

Ibbotson, R. (2007). Hitec HSR-8498HB Digital Servo Operation and Interface, Available from: http://robosavvy.com/Builders/i-Bot/HSR8498HB\%20 Servo.pdf Accessed: 2010-10-09

Freescale Semiconductor, MCF52235 ColdFire Integrated Microcontroller Product Brief, Available from: http://cache.freescale.com/files/32bit/doc/prod_brief/MCF5 2235PB.pdf?fsrch=1\&sr=4 Accessed: 2010-08-16

Liberty, J. \& MacDonald, B. (2008), Learning C\# 3.0, O'Reilly Media, ISBN: 978-0596521066, USA

Everett, H.R. (1995), Sensors for Mobile Robots, Theory and Applications, AK Peters, Ltd., ISBN: 1-56881-048-2, USA

*** (2007), HSR Serial Communication, Available from: www.staff.uni-bayreuth.de/ btp918/cmt2007/geraete/ hitec_hsr/ HSR_Serial_communication.pdf Accesed: 2009$12-26$ 